\title{
Explorando las Conceptualizaciones de la Pendiente en Estudiantes Universitarios
}

\section{Exploring Slope's Conceptualizations of University Students}

\author{
Martha Iris Rivera López * \\ ORCID iD 0000-0001-5597-0758 \\ Gerardo Salgado Beltrán ${ }^{* *}$ \\ ORCID iD 0000-0001-8133-5440 \\ Crisólogo Dolores Flores ${ }^{* * *}$ \\ ORCID iD 0000-0002-2748-6042
}

\begin{abstract}
Resumen
Este escrito reporta las conceptualizaciones de la pendiente de 21 estudiantes universitarios. Para la recolección de datos se utilizó una entrevista basada en tareas y para su análisis se identificaron las frases y procedimientos claves relacionados con las once conceptualizaciones reportadas por investigadores en educación matemática sobre el concepto de pendiente. Los estudiantes evidenciaron de una a ocho conceptualizaciones, entre las cuales se identificaron: Propiedad Física, Razón Algebraica, Propiedad Determinante, Constante Lineal, Coeficiente Paramétrico, Razón Geométrica, Indicador de Comportamiento y Situación Mundo Real (Situación Física).
\end{abstract}

Palabras clave: Conceptualizaciones. Pendiente. Estudiantes Universitarios. Entrevista Basada en Tareas.

\begin{abstract}
This article reports an investigation that explored Slope's conceptualizations in 21 university students. The data was collected with a Task-Based Interview and for its data analysis it focused on the identification of key phrases and procedures that referred to any of the eleven conceptualizations reported on the slope concept. The students evidenced from one to eight conceptualizations, among which were identified: Physical Property, Algebraic Ratio, Determining Property, Linear Constant, Parametric Coefficient, Geometric Ratio, Behavior Indicator, and Real-World Situation (Physical situation).
\end{abstract}

Keywords: Conceptualizations. Slope. University Students. Task-Based Interview.

\footnotetext{
* M.C. en Matemática Educativa, Universidad Autónoma de Guerrero (UAGro), Unidad Académica de Matemáticas. Estudiante de Doctorado en Matemática Educativa, Chilpancingo de los Bravo, Guerrero, México. Dirección postal: Av. Lázaro Cárdenas S/N, Ciudad Universitaria Chilpancingo, Gro. CP. 39087. E-mail: irivera@uagro.mx

** M.C. en Matemática Educativa, Universidad Autónoma de Guerrero (UAGro), Unidad Académica de Matemáticas. Estudiante de Doctorado en Matemática Educativa, Chilpancingo de los Bravo, Guerrero, México. Dirección postal: Av. Lázaro Cárdenas S/N, Ciudad Universitaria Chilpancingo, Gro. CP. 39087. E-mail: gerardosalgadobeltran@yahoo.es

*** Dr. en Matemática Educativa, Instituto Superior Pedagógico "Enrique J. Varona" de la Habana Cuba. Catedrático e investigador del Centro de Investigación en Matemática Educativa (CIMATE), Chilpancingo de los Bravo, Guerrero, México. Dirección postal: Av. Lázaro Cárdenas S/N, Ciudad Universitaria Chilpancingo, Gro. CP. 39087. E-mail: cdolores2@gmail.com.
} 


\section{Introducción}

La importancia del concepto de pendiente en la Educación Matemática se debe a que es necesaria para comprender y describir el comportamiento de funciones (TEUSCHER; REYS, 2010). El comprender este concepto, así como la linealidad, son clave para describir funciones no lineales, por ejemplo, cuadrática y exponencial (YERUSHALMY, 1997). Más aún, el concepto de pendiente es fundamental para comprender el concepto de derivada en Cálculo (CHRISTENSEN; THOMPSON, 2012; STANTON; MOORE-RUSSO, 2012; STROUP, 2002).

Por otro lado, este concepto se puede representar de diferentes maneras: algebraicamente, en fórmulas y ecuaciones; geométricamente, en gráficos; trigonométricamente, como la tangente del ángulo de inclinación de una recta, en cálculo como límite etc. Esto lo hace un concepto protagonista en el currículo matemático y fundamental para desarrollar un pensamiento matemático avanzado (CARLSON; OEHRTMAN; ENGELKE, 2010; CONFREY; SMITH, 1995; NOBLE et al., 2001). Su comprensión no es tarea fácil, ya que va más allá de solo considerarlo como un cálculo algebraico relacionado a la inclinación de una recta (LOBATO; SIEBERT, 2002). Además, es útil porque contribuye a la comprensión de fenómenos de la vida real (STUMP, 2001).

El uso de las representaciones de la pendiente, de manera independiente, no es suficiente para posibilitar su entendimiento, sino también su conexión entre ellas (NAGLE; MOORE-RUSSO, 2013a). Algunas investigaciones (ASIALA et al., 1997; BAKER; COOLEY; TRIGUEROS, 2000; BORJI; ALAMOLHODAEI; RADMEHR， 2018; FUENTEALBA et al., 2017) han reportado que los estudiantes universitarios suelen tener dificultades cuando se enfrentan a preguntas que involucran a la pendiente en diferentes representaciones, debido a que no han entendido este concepto ni trabajado a profundidad antes de ingresar a la Universidad (BORJI et al., 2018).

Las diversas maneras en que puede conceptualizarse la pendiente es una de las fuentes de errores y dificultades en estudiantes de diferentes niveles educativos (CHO; NAGLE, 2017). Los errores más comunes se dan en el cálculo de la pendiente por medio de la expresión algebraica $\frac{x_{2}-x_{1}}{y_{2}-y_{1}}$ y en su interpretación como $\frac{\text { run }}{\text { rise }}$ en la gráfica de una recta (BARR, 1980, 1981; CHO; NAGLE, 2017; SCHOENFELD; SMITH; ARCAVI, 1993).

Algunas dificultades surgen: al interpretar funciones lineales y sus gráficas (MOSCHKOVICH, 1990; ZASLAVSKY; SELA; LERON, 2002); en la conexión entre la 
expresión algebraica de una función lineal y su representación gráfica (BIRGIN, 2012; HATTIKUDUR et al., 2012) y; cuando relacionan la pendiente con la razón de cambio o al interpretarla en situaciones funcionales del mundo real (DOLORES; GARCÍA; GÁLVEZ, 2017; DOLORES; RIVERA; GARCÍA, 2018; BILLINGS; KLANDERMAN, 2000; HERBERT; PIERCE, 2008; PLANINIC et al., 2012; TEUSCHER; REYS, 2010, 2012).

Stump (1999, 2001) y Moore-Russo, Conner y Rugg (2011) han categorizado las múltiples representaciones de la pendiente y las han denominado conceptualizaciones de la pendiente. En un primer estudio, Stump (1999) identificó siete conceptualizaciones en profesores de $9^{\circ}$ al $12^{\circ}$ grado: Razón Algebraica (A), Razón Geométrica (G), Propiedad Funcional (F), Propiedad Física (P), Conceptualización Trigonométrica (T), Coeficiente Paramétrico (PC) y Conceptualización en Cálculo (C). Posteriormente, Stump (2001) agrega, Situación Mundo Real (R), la cual la clasifica en situaciones físicas (RP) y situaciones funcionales (RF). Y finalmente, Moore-Russo, Conner y Rugg (2011) identifican tres conceptualizaciones más: Indicador de Comportamiento (B), Constante Lineal (L) y Propiedad Determinante (D), tal como se describen en el Cuadro 1.

\begin{tabular}{|c|c|}
\hline CONCEPTUALIZACIÓN & DESCRIPCIÓN \\
\hline Razón Algebraica & $\begin{array}{l}\text { Cambio en } y \text { entre cambio en } x \text {, razón con la expresión algebraica } \frac{y_{2}-y_{1}}{x_{2}-x_{1}} \mathrm{o} \\
\frac{\Delta y}{\Delta x}\end{array}$ \\
\hline Razón Geométrica & $\begin{array}{l}\text { La razón del desplazamiento vertical y desplazamiento horizontal en la } \\
\text { gráfica de una recta. Subida sobre corrida en el gráfico de una recta. }\end{array}$ \\
\hline Propiedad Funcional & $\begin{array}{l}\text { Razón de cambio constante entre dos variables encontradas en } \\
\text { representaciones múltiples, incluyendo tablas y descripciones verbales (por } \\
\text { ej. cuando } x \text { incrementa en } 2, y \text { incrementa en } 3 \text { ). }\end{array}$ \\
\hline Situación Mundo-Real & $\begin{array}{l}\text { Situación física (estática, por ej.: una rampa, escalera etc.) o situación } \\
\text { funcional (dinámica, por ej.: distancia en función del tiempo, volumen en } \\
\text { función del tiempo etc.) }\end{array}$ \\
\hline $\begin{array}{l}\text { Indicador de } \\
\text { comportamiento }\end{array}$ & $\begin{array}{l}\text { Número real con signo que indica crecimiento }(+) \text {, decrecimiento }(-) \text {, } \\
\text { tendencia horizontal de la línea }(0) \text {. Si no es cero, indica la intersección con } \\
\text { el eje } x \text {. }\end{array}$ \\
\hline Propiedad Física & $\begin{array}{l}\text { Descripción de una recta utilizando expresiones como grado, inclinación, } \\
\text { tendencia, ladeo, declive, ángulo, etc. }\end{array}$ \\
\hline Coeficiente Paramétrico & Coeficiente $m$ (o su valor numérico) en $y=m x+b$ ó $y-y_{1}=m\left(x-x_{1}\right)$. \\
\hline Trigonométrica & $\begin{array}{l}\text { Propiedad relacionada con el ángulo de una recta que hace con una recta } \\
\text { horizontal; tangente del ángulo de inclinación. }\end{array}$ \\
\hline En Cálculo & $\begin{array}{l}\text { Medida relacionada con la derivada como la pendiente de la tangente a una } \\
\text { curva, de una recta secante, o cómo razón de cambio instantánea para } \\
\text { cualquier función (incluso una no lineal). }\end{array}$ \\
\hline Propiedad Determinante & $\begin{array}{l}\text { Propiedad que determina si las rectas son paralelas o perpendiculares entre } \\
\text { sí; además de determinar una recta si da un punto y su pendiente. }\end{array}$ \\
\hline Constante Lineal & $\begin{array}{l}\text { Propiedad constante y única para las rectas; pendiente de la recta no es } \\
\text { afectada por la traslación. Propiedad constante que garantiza la colinealidad }\end{array}$ \\
\hline
\end{tabular}




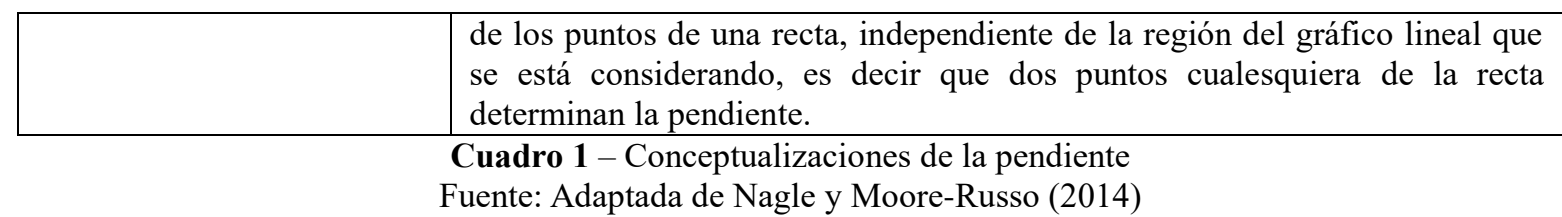

Las conceptualizaciones de la pendiente se han constituido como un eje referencial para otros estudios que involucran profesores y/o estudiantes (por ej.: CASEY; NAGLE, 2016; MUDALY; MOORE-RUSSO, 2011; NAGLE; MOORE-RUSSO, 2013a; NAGLE; MOORE-RUSSO; MARTÍNEZ-PLANELL 2016; NAGLE; MOORE-RUSSO; STYERS, 2017), el currículo (NAGLE; MOORE-RUSSO, 2014; STANTON; MOORE-RUSSO, 2012) y los libros de texto (KIM, 2012).

Sin embargo, hay vacíos y necesidades por satisfacer en esta línea de investigación. En este sentido, Stanton y Moore-Russo (2012) sugieren estudiar las conceptualizaciones de pendiente que ponen en juego estudiantes de diferentes niveles académicos de otros países para complementar las existentes y aportar nuevos elementos para la comprensión de la pendiente, ya que en la última década la mayoría de los estudios sobre esta línea de investigación acerca del concepto de pendiente se han realizado en el contexto estadounidense.

En México, el estudio de las conceptualizaciones de la pendiente es reciente y escasa. Algunos trabajos de investigación se han centrado en estudiar la razón de cambio en estudiantes (DOLORES; ALARCÓN; ALBARRAN, 2002; DOLORES; GARCÍA; GÁLVEZ, 2017). Dolores, Rivera y García (2018) reportó que la enseñanza-aprendizaje de la pendiente prioriza lo procedimental y el desarrollo de nociones conceptuales queda relegado a un segundo plano. Sus resultados le permitieron plantear la hipótesis de que se debe a la limitada comprensión acerca de la razón de cambio y su vínculo con la pendiente, tanto en profesores como en estudiantes.

Esto ha motivado, a continuar la línea de investigación de Stanton y Moore-Russo (2012) y plantearnos como pregunta de investigación: ¿Qué conceptualizaciones de la pendiente tienen los estudiantes universitarios? Y para responderla, asumimos como objetivo: identificar las conceptualizaciones de la pendiente que evidencian al discutir y resolver tareas relacionadas con este concepto.

\section{Metodología}

Esta investigación es cualitativa y exploratoria, ya que interesa identificar las 
conceptualizaciones de la pendiente que ponen en juego los estudiantes al resolver tareas que involucran al concepto. Para la recolección de datos se utilizó una entrevista basada en tareas, en el sentido de Goldin (2000). Consideramos que ésta es apropiada, ya que permite conocer las ideas y procedimientos matemáticos que utilizan los estudiantes al resolver tareas.

Una Entrevista Basada en Tareas puede ser estructurada o semiestructurada y requiere de una interacción mínima entre un sujeto (el que resuelve la tarea) y un entrevistador (el que pregunta), donde el sujeto habla durante, o inmediatamente después, de resolver una tarea (pregunta, problema, actividad) evidenciando, así, su conocimiento, su comportamiento y razonamiento en la resolución de la tarea (KOICHU; HAREL, 2007). Para identificar las conceptualizaciones de la pendiente que evidencian estudiantes universitarios se utilizó una Entrevista Basada en Tareas semiestructurada y las once conceptualizaciones de la pendiente descritas en el Cuadro 1.

\subsection{Participantes}

Los participantes fueron 21 estudiantes (doce hombres y nueve mujeres), cuyas edades varían entre diecisiete y dieciocho años. Recientemente, habían culminado sus estudios del Preuniversitario en diversos Subsistemas Educativos ubicados en diferentes regiones del Estado de Guerrero, México. Todos obtuvieron un promedio en matemáticas mayor a 8 y habían tomado los cursos de Geometría Analítica y Cálculo. Todos estaban legalmente inscritos a la Licenciatura en Matemáticas y se encontraban tomando un curso propedéutico introductorio, el cual tenía una duración de 8 horas diarias durante cuatro semanas.

\subsection{Diseño del instrumento y validación}

Las tareas que integran el protocolo empleado en esta investigación son el resultado del trabajo en conjunto de los investigadores de este estudio. Para su elaboración se consideraron los resultados de diversas investigaciones (MUDALY; MOORE-RUSSO, 2011; NAGLE et al., 2013; STUMP, 1999, 2001) que han explorado las conceptualizaciones de la pendiente en estudiantes y/o profesores.

También, se revisaron cinco libros de texto sugeridos en documentos oficiales del Preuniversitario (SALAZAR, 2010; CUELLAR, 2010; MAZÓN, 1997; MORA; RÍO, 2009; CONTRERAS et al., 2009). La revisión de los libros se debió a que es uno de los antecedentes académicos inmediatos que han sido pieza fundamental en la formación de los 
estudiantes que aspiran a realizar sus estudios universitarios. Permitió a los investigadores conocer la demanda cognitiva requerida para trabajar el concepto de la pendiente en ese Nivel Educativo. Esto fue de suma importancia para el diseño de las tareas, dado que los participantes son de recién ingreso a la universidad y en el momento de la recolección de datos, no habían tomado un curso de matemática superior. Esto ayudó que las tareas diseñadas fueran asequibles para los estudiantes.

El protocolo inicial se conformó por trece tareas. Para ganar rigor y confiabilidad en los datos se validó con diez estudiantes que se encontraban en $12^{\circ}$ grado del Preuniversitario. Para la validación también se utilizó la Entrevista Semiestructurada Basada en Tareas, cada entrevista estuvo a cargo de un investigador, misma que fue videograbada para su posterior análisis. En esta fase, el entrevistador planteó la tarea y pidió al estudiante platicar sus ideas y procedimientos empleados durante la resolución. En esta interacción, si el entrevistador detectaba una frase o procedimiento clave para los objetivos del estudio, realizó preguntas auxiliares como: ¿por qué lo hiciste así?, ¿conoces otra vía de solución?, ¿a qué te refieres con este término?, ¿por qué utilizaste esa fórmula? etc., con la intención de profundizar en los razonamientos empleados.

La validación permitió discriminar y asegurar que las tareas fueran congruentes con el objetivo, así como recoger el nivel de partida requerido para su resolución. De esta manera, se prescindió de dos tareas y el refinamiento en el planteamiento de 8. De modo que, el protocolo final se integró por once tareas que involucran diversas representaciones de la pendiente (ver anexo), de las cuales una fue adaptada de Mudaly y Moore-Russo (2011) y dos de Stump (1999).

\subsection{Recolección de Datos}

Los datos fueron recolectados en las instalaciones de la Unidad Académica de Matemáticas adjunta a la Universidad Autónoma de Guerrero, en la ciudad de Chilpancingo de los Bravo, México. Las entrevistas se realizaron en tres cubículos por los tres investigadores involucrados en el estudio. Cada entrevista tuvo en promedio una duración de 90 minutos. Fueron videograbadas (con previa autorización de los participantes) con la finalidad de captar: evidencia escrita, movimientos kinestésicos empleados, evidencia verbal etc. La entrevista giró en torno al protocolo proporcionado al estudiante y preguntas auxiliares (¿por qué lo hiciste así?, ¿conoces otra vía de solución?, ¿a qué te refieres con este término?, ¿por qué utilizaste esa fórmula?), a fin de conocer a detalle su razonamiento. 


\subsection{Análisis de Datos}

Para el análisis de los datos se digitalizaron las producciones escritas y transcribieron las entrevistas en el procesador de texto (Microsoft Word). La Figura 1 muestra el diseño de la tabla que se utilizó para registrar el análisis individual de las producciones de los estudiantes. En ella, se registró con una $X$ la conceptualización identificada en cada tarea.

\begin{tabular}{|c|c|c|c|c|c|c|c|c|c|c|c|c|}
\hline Estudiante & \multicolumn{12}{|c|}{ Conceptualizaciones } \\
\hline \multirow{2}{*}{ Tareas $\left(T_{n}\right)$} & \multirow{2}{*}{ A } & \multirow{2}{*}{ G } & \multirow{2}{*}{$\mathrm{F}$} & \multicolumn{2}{|c|}{$\mathrm{R}$} & \multirow{2}{*}{$\mathrm{B}$} & \multirow{2}{*}{$\mathrm{P}$} & \multirow{2}{*}{$\mathrm{PC}$} & \multirow{2}{*}{$\mathrm{T}$} & \multirow{2}{*}{$\mathrm{C}$} & \multirow{2}{*}{$\mathrm{D}$} & \multirow{2}{*}{$\mathrm{L}$} \\
\hline & & & & $\mathrm{R}_{\mathrm{P}}$ & $\mathrm{R}_{\mathrm{F}}$ & & & & & & & \\
\hline $\mathrm{T}_{1}$ & & & & & & & & & & & & \\
\hline $\mathrm{T}_{2}$ & & & & & & & & & & & & \\
\hline $\mathrm{T}_{3}$ & & & & & & & & & & & & \\
\hline$\vdots$ & & & & & & & & & & & & \\
\hline $\mathrm{T}_{11}$ & & & & & & & & & & & & \\
\hline
\end{tabular}

Figura 1 - Ejemplo de la tabla empleada para el análisis de los datos por estudiante Fuente: Elaborada por los autores

Para registrar el análisis individual, cada investigador revisó y contrastó la evidencia digitalizada y las transcripciones de las entrevistas, a fin de identificar frases o procedimientos empleados por los estudiantes en cada tarea. De este modo, estableció códigos asociados a la descripción de cada conceptualización de la pendiente señalada en el Cuadro 1.

Para determinar las conceptualizaciones de la pendiente que manifiestan los estudiantes, los investigadores se reunieron y en conjunto, compararon y discutieron las codificaciones propuestas por cada uno. En caso de algún desacuerdo, se discutieron las posturas hasta llegar un consenso y decidieron el tipo de conceptualización presente. Esto permitió la confiabilidad de los resultados y garantizó su validez, credibilidad y rigor, eliminando, así, el sesgo de un único investigador.

\section{Resultados}

De los análisis y la triangulación realizada por los investigadores, se determinaron los códigos asociados a la descripción de cada conceptualización, los cuales se muestran en el Cuadro 2, permitiendo, así, la categorización. 


\begin{tabular}{|c|c|}
\hline CONCEPTUALIZACIÓN & DESCRIPCIÓN DE LOS CÓDIGOS \\
\hline Propiedad Física $(P)$ & $\begin{array}{l}\mathrm{P}_{1} \text { : Se asume el valor de la pendiente como el ángulo de inclinación. } \\
\mathrm{P}_{2} \text { : La pendiente es descrita empleando términos como: empinamiento, } \\
\text { inclinación o grado de inclinación. } \\
\mathrm{P}_{3} \text { : Emplea algún brazo o algún objeto (lápiz, regla, cuaderno, etc.) para } \\
\text { simular la inclinación. }\end{array}$ \\
\hline Razón Algebraica $(A)$ & $\begin{array}{l}\mathrm{A}_{1}: \text { Utiliza la fórmula } \frac{y_{2}-y_{1}}{x_{2}-x_{1}} \text { para calcular la pendiente a partir de las } \\
\quad \text { coordenadas de dos puntos en el plano. } \\
\mathrm{A}_{2}: \text { Cuantifica y utiliza los cambios } \Delta x \text { y } \Delta y \text { para obtener la pendiente. } \\
\mathrm{A}_{3} \text { : Calcula diferencias y obtiene la razón de cambio. }\end{array}$ \\
\hline Propiedad Determinante $(D)$ & $\mathrm{D}_{1}$ : Rectas paralelas tienen la misma pendiente. \\
\hline $\begin{array}{c}\text { Coeficiente Paramétrico } \\
(P C)\end{array}$ & $\begin{array}{l}\mathrm{PC}_{1}: \text { El valor numérico de la pendiente está dado por el número que acompaña } \\
\text { la } x \text { en la ecuación } y=m x+b \text {. } \\
\mathrm{PC}_{2} \text { : El valor numérico de la pendiente es el valor de } m \text { en } y-y_{1}= \\
m\left(x-x_{1}\right) \text {. } \\
\mathrm{PC}_{3} \text { : la pendiente en la ecuación de la forma } a x+b y+c=0 \text { es } m=-\frac{a}{b}\end{array}$ \\
\hline $\begin{array}{l}\text { Situación Mundo Real- } \\
\text { Situación Física }\left(R_{P}\right)\end{array}$ & $\begin{array}{l}\mathrm{R}_{\mathrm{P} 1} \text { : La pendiente es la subida o bajada. } \\
\mathrm{R}_{\mathrm{P} 2} \text { : La pendiente es un techo inclinado. }\end{array}$ \\
\hline Constante Lineal $(L)$ & $\begin{array}{l}\mathrm{L}_{1} \text { : Al trasladar una recta en el plano la pendiente se conserva. } \\
\mathrm{L}_{2} \text { : Dada la gráfica de una recta se puede tomar cualquier pareja de puntos y la } \\
\text { pendiente será la misma. }\end{array}$ \\
\hline Razón Geométrica $(G)$ & $\begin{array}{l}\mathrm{G}_{1}: \text { Representa la pendiente de la recta a través de aumento en } y \text { cuando } x \\
\text { aumenta su valor en } 1 . \\
\mathrm{G}_{2} \text { : Representa la pendiente de la recta a través de desplazamientos verticales } \\
\text { y horizontales. }\end{array}$ \\
\hline $\begin{array}{c}\text { Indicador de } \\
\text { Comportamiento }(B)\end{array}$ & $\begin{array}{l}\mathrm{B}_{1} \text { : Si la pendiente es positiva la recta es creciente y si es negativa entonces es } \\
\text { decreciente. } \\
\mathrm{B}_{2} \text { : La pendiente es cero si la recta es constante. }\end{array}$ \\
\hline
\end{tabular}

Cuadro 2 - Códigos asignados a la descripción de cada conceptualización Fuente: Elaborada por los autores

En el Cuadro 3 se muestran los códigos asociados a las conceptualizaciones evidenciadas por los estudiantes en cada tarea. Los nombres utilizados son pseudónimos que permiten identificar a cada estudiante.

\begin{tabular}{|c|c|c|c|c|c|c|c|c|c|c|c|}
\hline \multirow[t]{2}{*}{ Estudiante } & \multicolumn{11}{|c|}{ Tareas } \\
\hline & $T_{1}$ & $\mathbf{T}_{2}$ & $\mathbf{T}_{3}$ & $\mathbf{T}_{4}$ & $T_{5}$ & $T_{6}$ & $\mathbf{T}_{7}$ & $T_{8}$ & $T_{9}$ & $T_{10}$ & $\mathbf{T}_{11}$ \\
\hline Emanuel & $\mathrm{A}_{1}$ & $\mathrm{P}_{1}$ & $\mathrm{D}_{1}$ & & $\mathrm{P}_{1}$ & $\mathrm{P}_{1}, \mathrm{P}_{3}$ & $\mathrm{~A}_{3}$ & $\mathrm{~B}_{2}$ & & $\mathrm{P}_{1}$ & $\mathrm{P}_{1}$ \\
\hline Diana & $\mathrm{A}_{1}$ & $\begin{array}{l}\mathrm{P}_{2}, \\
\mathrm{P}_{3}\end{array}$ & & $\mathrm{~L}_{2}$ & $\mathrm{P}_{1}$ & $\mathrm{P}_{1}$ & $\mathrm{~A}_{3}$ & $\mathrm{~B}_{2}$ & $\mathrm{PC}_{1}$ & $\mathrm{P}_{1}$ & $\mathrm{P}_{2}$ \\
\hline Lorenzo & $\mathrm{A}_{1}$ & $\mathrm{P}_{1}$ & & & $\mathrm{P}_{1}$ & $\mathrm{R}_{\mathrm{P} 1}$ & $\mathrm{~A}_{3}$ & & & $\mathrm{P}_{1}$ & $\mathrm{P}_{1}$ \\
\hline Yareli & $\begin{array}{c}\mathrm{PC}_{1}, \mathrm{PC}_{2}, \\
\mathrm{PC}_{3}, \mathrm{G}_{2}\end{array}$ & $\mathrm{D}_{1}$ & $\mathrm{PC}_{2}$ & $\mathrm{PC}_{1}$ & $\mathrm{P}_{1}, \mathrm{P}_{3}$ & & $\mathrm{~A}_{3}$ & $\mathrm{~B}_{2}$ & $\begin{array}{c}\mathrm{PC}_{1}, \\
\mathrm{~B}_{1}\end{array}$ & $\mathrm{P}_{1}$ & $\mathrm{~L}_{1}$ \\
\hline Miguel & $\mathrm{A}_{1}, \mathrm{G}_{1}$ & $\mathrm{D}_{1}$ & $\begin{array}{l}\mathrm{G}_{1}, \\
\mathrm{D}_{1}\end{array}$ & $\mathrm{PC}_{1}$ & $\mathrm{P}_{1}$ & $\mathrm{P}_{1}, \mathrm{P}_{3}$ & $\mathrm{~A}_{3}$ & $\mathrm{~B}_{2}$ & $\mathrm{PC}_{1}$ & $\mathrm{P}_{1}$ & $\overline{D_{1}, L_{1}}$ \\
\hline Francisco & $\mathrm{A}_{1}$ & $\begin{array}{l}\mathrm{P}_{2}, \\
\mathrm{P}_{3}\end{array}$ & $\mathrm{~A}_{1}$ & $\mathrm{PC}_{1}$ & $\mathrm{P}_{2}, \mathrm{P}_{3}$ & $\mathrm{R}_{\mathrm{P} 1}$ & $\mathrm{~A}_{3}$ & $\mathrm{P}_{2}$ & $\overline{A_{1}}$ & $\mathrm{~A}_{1}$ & $\mathrm{~A}_{1}$ \\
\hline Armando & $\mathrm{A}_{1}$ & $\mathrm{P}_{1}$ & & & $\mathrm{P}_{1}$ & $\mathrm{R}_{\mathrm{P} 1}$ & $\mathrm{~A}_{3}$ & & & $\mathrm{P}_{1}$ & \\
\hline Ilse & $\mathrm{A}_{1}$ & $\mathrm{P}_{1}$ & & & $\mathrm{P}_{1}$ & $\mathrm{P}_{1}$ & & $\mathrm{~B}_{2}$ & & & $\mathrm{P}_{1}, \mathrm{P}_{3}$ \\
\hline Azurym & $\mathrm{A}_{1}, \mathrm{PC}_{1}, \mathrm{~L}_{2}$ & $\mathrm{D}_{1}$ & $\mathrm{D}_{1}$ & $\mathrm{PC}_{1}$ & $\mathrm{P}_{1}$ & $\mathrm{P}_{2}, \mathrm{P}_{3}$ & & $\mathrm{~B}_{2}$ & $\mathrm{PC}_{1}$ & $\mathrm{P}_{1}$ & $\mathrm{P}_{1}$ \\
\hline Josué & & & & & & $\mathrm{P}_{2}, \mathrm{P}_{3}$ & $\mathrm{~A}_{3}$ & & & & \\
\hline Alejandra & $\mathrm{A}_{1}$ & $D_{1}$ & $\mathrm{~A}_{1}$ & $\mathrm{PC}_{1}$ & $\mathrm{P}_{1}$ & $\mathrm{P}_{1}$ & $\mathrm{~A}_{3}$ & $\mathrm{P}_{1}$ & & $\mathrm{P}_{1}$ & $\mathrm{P}_{1}$ \\
\hline José & $\mathrm{A}_{1}$ & & $\mathrm{PC}_{2}$ & $A_{1}$ & $\mathrm{P}_{1}$ & $\mathrm{P}_{1}$ & $\mathrm{~A}_{3}$ & $\mathrm{P}_{2}$ & $\mathrm{PC}_{1}$ & $\mathrm{P}_{1}$ & $D_{1}$ \\
\hline Juan & $\mathrm{A}_{2}$ & $\mathrm{D}_{1}$ & & $\mathrm{PC}_{1}$ & $\mathrm{P}_{1}$ & $\mathrm{R}_{\mathrm{P} 1}$ & $\mathrm{~A}_{3}$ & $\mathrm{P}_{2}$ & & & $\mathrm{P}_{1}$ \\
\hline Axel & & & & & & $\mathrm{P}_{2}, \mathrm{P}_{3}$ & $\mathrm{~A}_{3}$ & & & $\mathrm{P}_{1}$ & $\mathrm{P}_{1}$ \\
\hline
\end{tabular}




\begin{tabular}{|c|c|c|c|c|c|c|c|c|c|c|c|}
\hline Andrea & & $\mathrm{P}_{1}$ & & & $\mathrm{P}_{1}$ & $\mathrm{P}_{1}$ & $\mathrm{~A}_{3}$ & & & $\mathrm{P}_{1}$ & \\
\hline Alán & $\mathrm{A}_{1}$ & $\mathrm{D}_{1}$ & $\mathrm{~A}_{1}$ & $\mathrm{PC}_{1}$ & $\begin{array}{c}\mathrm{P}_{1}, \\
\mathrm{P}_{2}, \mathrm{P}_{3}\end{array}$ & $\mathrm{R}_{\mathrm{P} 1}$ & $\mathrm{~A}_{3}$ & $\begin{array}{c}\mathrm{P}_{1}, \\
\mathrm{P}_{2}\end{array}$ & & $\mathrm{P}_{1}$ & $\mathrm{D}_{1}, \mathrm{~L}_{1}$ \\
\hline Adelina & & & & & & & & & & $\mathrm{P}_{1}$ & \\
\hline Elena & $\mathrm{P}_{1}$ & $\mathrm{P}_{1}$ & & & $\mathrm{P}_{1}$ & $\mathrm{P}_{2}, \mathrm{P}_{3}$ & & $\mathrm{P}_{1}$ & & & $\mathrm{P}_{1}$ \\
\hline Luis & & $\mathrm{R}_{\mathrm{P} 2}$ & & & & $\mathrm{R}_{\mathrm{P} 1}$ & $\mathrm{~A}_{3}$ & & & & $\mathrm{P}_{1}$ \\
\hline Alma & $\mathrm{A}_{1}$ & & & & & & & & & & \\
\hline Jesús & & & & & $\mathrm{P}_{1}$ & & & & & $\mathrm{P}_{1}$ & \\
\hline
\end{tabular}

Cuadro 3 - Códigos asociados a las producciones de los estudiantes por tarea Fuente: Elaborada por los autores

Para explicitar los elementos utilizados en la determinación de las conceptualizaciones de cada estudiante, tomamos como ejemplo el caso de Miguel, ya que evocó más conceptualizaciones en la resolución de las once tareas.

\subsection{Conceptualizaciones de la pendiente evidenciadas por Miguel}

Miguel evidenció siete conceptualizaciones de la pendiente: Razón Algebraica, Razón Geométrica, Propiedad Física, Coeficiente Paramétrico, Propiedad Determinante, Constante Lineal e Indicador de Comportamiento. Para fundamentar esta declaración a continuación se muestra el análisis realizado a sus producciones.

Para obtener la pendiente de una recta dada en un plano graduado, Miguel identifica las coordenadas cartesianas de dos puntos por donde pasa la recta y emplea la fórmula algebraica $m=\frac{y_{2}-y_{1}}{x_{2}-x_{1}}(A)$. Su respuesta la representó como desplazamientos verticales y horizontales en la gráfica $(G)$, tal como se observa en la Figura 2.

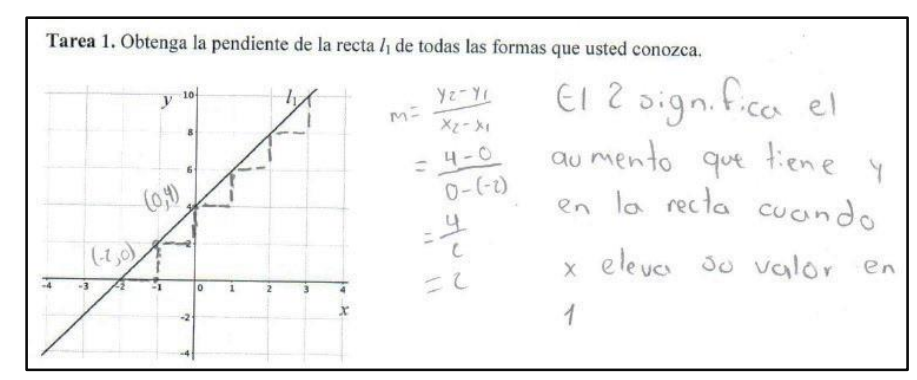

Figura 2 - Conceptualización Razón Algebraica y Razón Geométrica en los procedimientos de Miguel Fuente: Autores (2018)

Para la comparación de las pendientes de tres techos situados en una casa, Miguel se apoya del criterio de paralelismo, es decir: si dos o más rectas son paralelas entonces sus pendientes son iguales $(D)$. Este criterio también fue utilizado en la resolución de las tareas 3 y 5 para argumentar la posibilidad de que a partir de la gráfica de una recta existen otras que tienen la misma pendiente $(D)$, y argüir que la traslación de una recta en el plano no afecta el valor numérico de su pendiente $(L)$.

Por otra parte, para graficar una recta a partir de un punto dado y su pendiente 
(positiva), Miguel parte del punto e interpreta y representa la pendiente como aumento en $y$ producido por un aumento en $x(G)$. Para identificar una función lineal a partir de una tabla numérica, construye la expresión algebraica que describe el comportamiento de dichos valores y la compara con la forma de la ecuación de la recta $y=m x+b(P C)$.

Para la comparación y el establecimiento de la relación entre las pendientes de dos rectas dadas en planos no graduados, tarea 5 y 10, Miguel asume al ángulo de inclinación como la pendiente de una recta $(P)$. De manera que si una recta tiene mayor ángulo, entonces su pendiente es mayor (Figura 3) y que al duplicar su ángulo de inclinación entonces su pendiente se duplica. Esta postura persistió incluso cuando interpretó una señalización vial relacionada con la pendiente de un camino, en sus argumentos incorporó movimientos kinestésicos, usando sus brazos para describir una subida $(P)$.

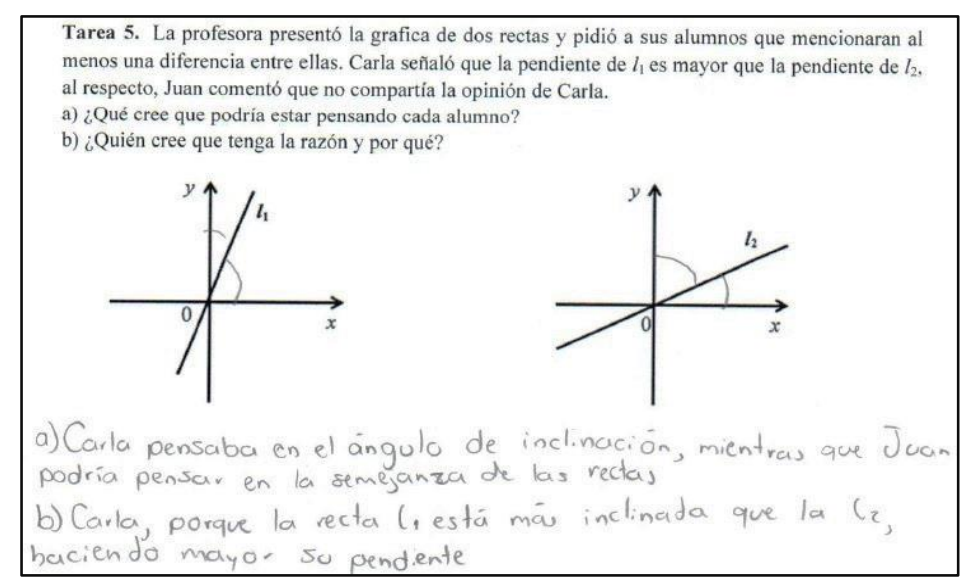

Figura 3 - Ejemplo de la Conceptualización Propiedad Física en los procedimientos de Miguel Fuente: Autores (2018)

Al resolver una tarea que involucraba una situación expresada en lenguaje común en la que se requería la identificación y el cálculo de una razón de cambio constante, Miguel realiza cálculo de un cociente de diferencias $(A)$ misma que asocia con una constante, pero no la interpreta como una razón de cambio.

Miguel no evidenció la conceptualización en Cálculo, ya que para él la pendiente es una característica propia de la recta y no de una curva (por ej., parábola). Esta idea la manifestó cuando se le presentaron cuatro gráficas (tres rectas y una parábola) y se le pidió que eligiera cuál de ellas podría tener una pendiente de 2 . Sus argumentos se centraron en que por la ubicación de las rectas en el plano estas tendrían pendientes positivas, negativas o cero (B). Tal como se muestra en el extracto de la entrevista.

Entrevistador: Para ti, ¿Cuáles de las gráficas podrían tener pendiente dos? Miguel: Para mí, ninguna.

Entrevistador: ¿Por qué?

Miguel: Uhmm, la primera tiene pendiente negativa y no puede ser dos.

Entrevistador: ¿Cómo sabes que es negativa? 
Miguel: Es que se ve que tiene un ángulo de inclinación mayor a noventa grados, esto hace que su pendiente sea negativa, la segunda tiene pendiente positiva su ángulo es menor a noventa grados, pero su pendiente supera a dos, la tercera no puede tener pendiente dos ya que es horizontal y por eso su pendiente es cero.

Entrevistador: ¿Y qué ocurre con la cuarta gráfica?

Miguel: La última no es una recta, que yo sepa la pendiente es para las rectas. No sé si podría sacarle la pendiente a ésta [señala con su lápiz la gráfica de la parábola] (Diálogo entre el entrevistador y Miguel, 2018).

De las producciones de Miguel se destacó que, al confrontarse con la gráfica de una recta, describe su pendiente en términos del ángulo de inclinación, mientras que cuando la información dada es la ecuación de la recta (Tarea 11), asocia la pendiente directamente con el número que acompaña la $x$ en $y=m x+b$. Finalmente, las conceptualizaciones que no evidenció fueron: Trigonométrica, Propiedad Funcional, Situación Mundo Real y la de Cálculo.

De manera general, se encontró que los estudiantes evidenciaron de una a ocho conceptualizaciones entre las cuales se identificaron: Propiedad Física, Razón Algebraica, Propiedad Determinante, Constante Lineal, Coeficiente Paramétrico, Razón Geométrica, Indicador de Comportamiento y Situación Mundo Real- Situación Física. En la Tabla 1 se muestra cuántas y cuáles conceptualizaciones se identificaron en cada estudiante. En esta aparece una $X_{n}$, donde $n$ representa el número de tareas donde fue identificada la conceptualización.

Tabla 1 - Conceptualizaciones de la pendiente en estudiantes universitarios

\begin{tabular}{|c|c|c|c|c|c|c|c|c|c|c|c|c|c|}
\hline \multirow[b]{2}{*}{ Estudiante } & \multicolumn{12}{|c|}{ Conceptualizaciones } & \multirow{2}{*}{$\begin{array}{c}\text { Núm. de } \\
\text { conceptualizaciones }\end{array}$} \\
\hline & $A$ & $G$ & $F$ & $R_{P}$ & $\begin{array}{l}R \\
R_{F}\end{array}$ & $B$ & $P$ & $P C$ & $T$ & $C$ & $D$ & $L$ & \\
\hline Emanuel & $\mathrm{X}_{2}$ & & & & & $\mathrm{X}_{1}$ & $\mathrm{X}_{5}$ & & & & $\mathrm{X}_{1}$ & & 4 \\
\hline Diana & $\mathrm{X}_{2}$ & & & & & $\mathrm{X}_{1}$ & $\mathrm{X}_{5}$ & $\mathrm{X}_{1}$ & & & & $\mathrm{X}_{2}$ & 5 \\
\hline Lorenzo & $\mathrm{X}_{2}$ & & & $\mathrm{X}_{1}$ & & & $\mathrm{X}_{4}$ & & & & & & 3 \\
\hline Yareli & $\mathrm{X}_{1}$ & $\mathrm{X}_{1}$ & & & & $\mathrm{X}_{2}$ & $\mathrm{X}_{2}$ & $\mathrm{X}_{3}$ & & & $\mathrm{X}_{1}$ & $\mathrm{X}_{1}$ & 7 \\
\hline Miguel & $\mathrm{X}_{2}$ & $\mathrm{X}_{2}$ & & & & $\mathrm{X}_{1}$ & $\mathrm{X}_{3}$ & $\mathrm{X}_{2}$ & & & $\mathrm{X}_{3}$ & $\mathrm{X}_{1}$ & 7 \\
\hline Francisco & $\mathrm{X}_{6}$ & & & $\mathrm{X}_{1}$ & & & $\mathrm{X}_{3}$ & $\mathrm{X}_{1}$ & & & & & 4 \\
\hline Armando & $\mathrm{X}_{2}$ & & & $\mathrm{X}_{1}$ & & & $\mathrm{X}_{3}$ & & & & & & 3 \\
\hline Ilse & $X_{1}$ & & & & & $\mathrm{X}_{1}$ & $\mathrm{X}_{4}$ & & & & & & 3 \\
\hline Azurym & $X_{1}$ & & & & & $\mathrm{X}_{1}$ & $\mathrm{X}_{4}$ & $\mathrm{X}_{3}$ & & & $\mathrm{X}_{2}$ & $\mathrm{X}_{1}$ & 6 \\
\hline Josué & $\mathrm{X}_{1}$ & & & & & & $\mathrm{X}_{1}$ & & & & & & 2 \\
\hline Alejandra & $\mathrm{X}_{3}$ & & & & & & $\mathrm{X}_{5}$ & $\mathrm{X}_{1}$ & & & $\mathrm{X}_{1}$ & & 4 \\
\hline José & $X_{3}$ & & & & & & $X_{4}$ & $\mathrm{X}_{2}$ & & & $\mathrm{X}_{1}$ & & 4 \\
\hline Juan & $\mathrm{X}_{2}$ & & & $\mathrm{X}_{1}$ & & & $\mathrm{X}_{3}$ & $\mathrm{X}_{1}$ & & & $\mathrm{X}_{1}$ & & 5 \\
\hline Axel & $X_{1}$ & & & & & & $X_{3}$ & & & & & & 2 \\
\hline Andrea & $\mathrm{X}_{1}$ & & & & & & $\mathrm{X}_{4}$ & & & & & & 2 \\
\hline Alán & $\mathrm{X}_{2}$ & & & $\mathrm{X}_{1}$ & & & $\mathrm{X}_{3}$ & $\mathrm{X}_{1}$ & & & $\mathrm{X}_{2}$ & $\mathrm{X}_{1}$ & 6 \\
\hline Adelina & & & & & & & $\mathrm{X}_{1}$ & & & & & & 1 \\
\hline Elena & & & & & & & $\mathrm{X}_{6}$ & & & & & & 1 \\
\hline Luis & $\mathrm{X}_{1}$ & & & $\mathrm{X}_{2}$ & & & $\mathrm{X}_{1}$ & & & & & & 3 \\
\hline Alma & $\mathrm{X}_{1}$ & & & & & & & & & & & & 1 \\
\hline Jesús & & & & & & & $\mathrm{X}_{2}$ & & & & & & 1 \\
\hline
\end{tabular}




\section{Discusión y Conclusiones}

Nuestros resultados describen que los estudiantes tienen un conocimiento limitado sobre las diversas representaciones de la pendiente, ya que aproximadamente el 52\% evidenciaron entre una y tres conceptualizaciones, de las cuales la Propiedad Física y la Razón Algebraica fueron las que más emplearon en la resolución de las tareas. Sólo el 9.5\% evidenciaron siete conceptualizaciones. Las conceptualizaciones Propiedad Funcional, Trigonométrica, Cálculo y Situación mundo real en su categoría de situaciones funcionales estuvieron ausentes en las producciones de todos los estudiantes.

El 95\% de los estudiantes utilizaron la pendiente como el ángulo de la inclinación de una recta. Cuando se involucraron en una tarea que requería de interpretar la subida de un camino en términos de su pendiente, a través de una señalización vial, utilizaron frases como empinamiento e inclinación como equivalentes a la pendiente, algunos realizaron movimientos kinestésicos, empleando sus brazos u objetos (lápiz, regla, cuaderno etc.).

Estos resultados son comprensibles, ya que según Cheng y Sabinin (2008) las personas comienzan a aprender acerca de la pendiente a través de la inclinación de objetos en la vida cotidiana, como: rampas, escaleras, resbaladillas, calles, cerros, techos de casas etc. De este modo, los estudiantes pueden visualizar el concepto matemático de pendiente sin depender de ninguna habilidad matemática, como usar una razón o calcular un límite (DENIZ; KABAEL, 2017; NAGLE; MOORE-RUSSO, 2013b). Sin embargo, el que los estudiantes interpreten la pendiente como el ángulo no debe considerarse una idea errónea (RASSLAN; VINNER, 1995), ya que este es un aspecto importante de la pendiente y de acuerdo con Zaslavsky et al. (2000) enseñar a los estudiantes a ignorarlo o tratarlo como irrelevante no contribuye a la comprensión del concepto.

El reconocimiento de la pendiente como Coeficiente Paramétrico se produjo en el $43 \%$ de los estudiantes al identificarla como $m$ en la ecuación de la recta $y=m x+b$ la cual referenciaron como: el número que acompaña a la $x$ en la ecuación, el coeficiente de $x$, la constante que acompaña a la $x$. Incluso, algunos hicieron referencia a su valor numérico como $m$ en la ecuación $y-y_{1}=m\left(x-x_{1}\right)$ y una minoría como $m=-\frac{a}{b}$ en la ecuación de la forma $a x+b y+c=0$. El 90\% de los estudiantes desconocían la interpretación geométrica del concepto, evidenciando una comprensión limitada del concepto de pendiente y su vínculo con una función lineal, resultado parecido a lo que reporta Birgin (2012).

Asimismo, la desconexión entre la pendiente y razón de cambio fue evidente en este 
grupo de estudiantes, ya que ninguno manifestó la conceptualización en Cálculo. Este resultado puede ser producto de su instrucción, probablemente porque sus profesores no propician este vínculo o los miran como conceptos aislados. Stump (1999) y Nagle et al. (2013) muestran resultados en profesores estadounidenses que respaldan nuestra hipótesis.

Asimismo, Dolores, Rivera y García (2018) en estudiantes de Bachillerato, y Teuscher y Reys (2012), en estudiantes de Cálculo Avanzado, encuentran la misma desconexión. Esto implicó que los estudiantes al confrontarse con una tarea que requería de reconocer si una parábola podía tener una pendiente de 2 , la totalidad de los estudiantes manifestaron que la pendiente solo se podía calcular en rectas.

Los participantes previamente habían sido instruidos en los cursos de Geometría Analítica y Trigonometría en el Preuniversitario, nivel académico en el cual los Programas de Estudio sugieren como punto de partida el tratamiento de la pendiente de una recta como la tangente de su ángulo de inclinación. Sin embargo, nuestros resultados evidenciaron que ninguno evocó la conceptualización Trigonométrica en sus procedimientos y argumentos. Esto nos hace suponer que en su instrucción existe una desconexión entre la definición de la pendiente como la tangente trigonométrica de su ángulo de inclinación y el cociente $\frac{y_{2}-y_{1}}{x_{2}-x_{1}}$. Este resultado en particular es similar a lo encontrado por Stump (2009) y Nagle y MooreRusso (2013b) con profesores.

Si bien, los criterios de paralelismo y perpendicularidad son un tema sugerido en los Programas de Estudio de Bachillerato (SEP, 2013), se esperaba que una porción significativa de estudiantes manifestara su reconocimiento y dominio al resolver algunas tareas que lo requerían. Sin embargo, solo el 38\% lo empleó exclusivamente para el caso de las rectas paralelas en un contexto gráfico. Ninguno de los estudiantes referenció el criterio para el caso de las rectas perpendiculares.

Nuestro estudio no solo reveló las conceptualizaciones de la pendiente ya reportadas, los procedimientos y argumentos de casi el 38\% de los estudiantes evidenciaron que la pendiente es conceptualizada como la magnitud de una recta, techo o calle. Este hallazgo es similar a lo encontrado en profesores de matemáticas por Byerley y Thompson (2017). Por otro lado, aproximadamente el $48 \%$ consideraron que la pendiente de una recta es la intersección de esta con algún eje coordenado ( $x$ o y), resultado que Birgin (2012) encontró con estudiantes turcos de octavo grado. También, se encontró que alrededor del 10\% de los estudiantes consideran que la pendiente de una recta es la recta misma (el objeto).

En conclusión, podemos decir que el estudio ha revelado un escaso conocimiento de 
las diversas representaciones de la pendiente por parte de los estudiantes, tal como también lo ha identificado Asiala et al. (1997) y Borji et al. (2018). Se percibió una desconexión entre los aspectos geométricos y variacionales de la pendiente, parece ser que su aprendizaje e instrucción se ha centrado en lo procedimental relegando a un segundo plano lo conceptual.

Por otra parte, el estudio también identificó una serie de errores y malentendidos sobre ecuaciones de función lineal, funciones crecientes, decrecientes, rectas paralelas y perpendiculares, gráficos y el concepto de pendiente. Algunos confundieron los aspectos algebraicos y geométricos de la pendiente y más aún desconocen los variacionales.

\section{Limitaciones e implicaciones}

Este estudio proporciona una visión crítica de las conceptualizaciones de la pendiente de los estudiantes universitarios mexicanos. Sin embargo, reconocemos que como en cualquier estudio hay limitaciones. Esta investigación está limitada por el tamaño de la muestra, en general, solo 21 estudiantes participaron en este estudio cualitativo, ya que un tamaño de muestra más grande habría permitido una recolección más robusta de datos y podríamos haber introducido aspectos cuantitativos.

Recomendamos realizar estudios similares en otros estados de la república mexicana, que involucren estudiantes y profesores de otros niveles educativos, la instrucción del profesor y los libros de texto, ya que es escasa la investigación en México sobre esta línea y, así, equiparar los resultados con lo reportado en otros países. De este modo, podríamos conocer la correlación que existe entre estos elementos que conforman el proceso de enseñanza-aprendizaje de la pendiente $\mathrm{y}$, por tanto, aportar elementos para mejorar su entendimiento.

\section{Referencias}

ASIALA, M.; COTTRILL, J.; DUBINSKY, E.; CHWINGENDORF, K. E. The development of students' graphical understanding of the derivative. Journal of Mathematical Behavior, v.16, n. 4, p. 399-430, 1997.

BAKER, B.; COOLEY, L.; TRIGUEROS, M. A Calculus Graphing Schema, Journal for Research in Mathematics Education, Barcelona, v. 31, n. 5, p. 557-576, 2000.

BARR, G. Graphs, Gradients and Intercepts. Mathematics in School, London, v. 9, n. 1, p. 5-6, 1980.

BARR, G. Some Student Ideas on the Concept of Gradient. Mathematics in School, London, v. 10, n. 1, p. 14-17, 1981. 
BILLINGS, E. M.; KLANDERMAN, D. Graphical representations of speed: obstacles preservice K-8 teachers experience. School Science and Mathematics, Hoboken, v. 100, n. 8, p. 440-450, 2000.

BIRGIN, O. Investigation of eighth-grade students' understanding of the slope of the linear function. Bolema, Rio Claro, v. 26, n. 42a, p. 139-162, 2012.

BORJI, V.; ALAMOLHODAEI, H.; RADMEHR, F. Application of the APOS-ACE Theory to Improve Students' Graphical Understanding of Derivative. Eurasia Journal of Mathematics, Science and Technology Education, Poznan, v.14, n.7, p. 2947-2967, 2018.

BORJI, V.; FONT, V.; ALAMOLHODAEI, H.; SÁNCHEZ, A. Application of the Complementarities of Two Theories, APOS and OSA, for the Analysis of the University Students' Understanding on the Graph of the Function and its Derivative. Eurasia Journal of Mathematics, Science and Technology Education, Poznan, v.14, n. 6, p. 2301-2315, 2018.

BYERLEY, C.; THOMPSON, P. Secondary mathematics teachers' meanings for measure, slope, and rate of change. The Journal of Mathematical Behavior, v. 48, p. 168- 193, 2017.

CARLSON, M.; OEHRTMAN, M.; ENGELKE, N. The precalculus concept assessment: A tool for assessing students' reasoning abilities and understandings. Cognition and Instruction, Maine, v. 28, n. 2, p. 113-145, 2010.

CASEY, S.; NAGLE, C. Students' use of slope conceptualizations when reasoning about the line of best fit. Educational Studies in Mathematics, New York, v. 92, n. 2, p. 163-177, 2016.

CHENG, D.; SABININ, P. Elementary students' conceptions of steepness. En: FIGUERAS, O; CORTINA, J. L.; ALATORRE, S.; ROJANO, T.; SEPÚLVEDA, A. (Ed.). Proceedings of the Joint Meeting of PME 32 and PME-NA XXX, México: Cinvestav-UMSNH, 2008. p. 297-304.

CHO, P.; NAGLE, C. Procedural and Conceptual Difficulties with Slope: An Analysis of Students' Mistakes on Routine Tasks. International Journal of Research in Education and Science, Taipei, v. 3, n. 1, p. 135-150, 2017.

CHRISTENSEN, W. M.; THOMPSON, J. R. Investigating graphical representations of slope and derivative without a physics context. Physical Review Special Topics-Physics Education Research, Ridge, v. 8, n. 2, p. 023101, 2012.

CONFREY, J.; SMITH, E. Splitting, covariation, and their role in the development of exponential functions. Journal for Research in Mathematics Education, Reston, v. 26, p. 66-86, 1995.

CONTRERAS, L.; MARTÍNEZ, M.; LUGO, O.; MONTES, M. Cálculo Diferencial e Integral, México: Santillana, 2009.

CUELLAR, J. Matemáticas III. México: McGraw Hill, 2010.

DENIZ, O.; KABAEL, T. Students' Mathematization Process of the Concept of Slope within the Realistic Mathematics Education. Hacettepe Üniversitesi Eğitim Fakültesi Dergisi (H.U. Journal of Education), Ankara, v. 32, n. 1, p. 123-142, 2017.

DOLORES, C.; ALARCÓN, G.; ALBARRÁN, D. Concepciones alternativas sobre las gráficas cartesianas del movimiento: el caso de velocidad y la trayectoria. Revista Latinoamericana de Investigación en Matemática Educativa, México, v. 5, n. 3, p. 225-250, 2002.

DOLORES, C.; GARCÍA, J.; GÁLVEZ, A. Estabilidad y cambio conceptual acerca de las razones de cambio en situación escolar. Educación Matemática, México, v. 29, n. 2, p. 125-158, 2017. 
DOLORES, C.; RIVERA, M. I.; GARCÍA, J. Exploring mathematical connections of pre-university students through tasks involving rates of change. International Journal of Mathematical Education in Science and Technology, London, UK, p. 1-21, 2018.

FUENTEALBA, C.; SÁNCHEZ-MATAMOROS, G.; BADILLO, E.; TRIGUEROS, M. Thematization of derivative schema in university students: Nuances in constructing relations between a function's successive derivatives. International Journal of Mathematical Education in Science and Technology, London, v. 48, n. 3, p. 374-392, 2017.

GOLDIN, G. A scientific perspective on structured, task-based interviews in mathematics education research. En: KELLY, A.; LESH, R. (Ed.). Handbook of Research Design in Mathematics and Science Education. New York: Routledge, 2000. p. 517-545.

HATTIKUDUR, S.; PRATHER, R.; ASQUITH, P.; KNUTH, E.; NATHAN, M.; ALIBALI, M. Constructing graphical representations: Middle schoolers' developing knowledge about slope and intercept. School Science and Mathematics, Hoboken, v. 112, n. 4, p. 230-240, 2012.

HERBERT, S.; PIERCE, R. An 'Emergent Model' for Rate of Change. International Journal of Computers for Mathematical Learning, Wayshesville, v. 13, n. 3, p. 231-49, 2008.

KIM, R. The quality of non-textual elements in mathematics textbooks: an exploratory comparison between South Korea and the United States. ZDM, Berlin, v. 44, n. 2, p. 175-187. 2012.

KOICHU, B.; HAREL, G. Triadic interaction in clinical task-based interviews with mathematics teachers. Educational Studies in Mathematics, New York, v. 65, n. 3, p. 349-365. 2007.

LOBATO, J.; SIEBERT, D. Quantitative reasoning in a reconceived view of transfer. The Journal of Mathematical Behavior, v. 21, p. 87-116, 2002.

MAZÓN, J. Calculo Diferencial. México: Mc Graw Hill, 1997.

MOORE-RUSSO, D.; CONNER, A.; RUGG, K. Can slope be negative in 3-space? Studying concept image of slope through collective definition construction. Educational Studies in Mathematics, New York, v. 76, n. 1, p. 3-21, 2011.

MORA, E.; RÍO, M. Cálculo Diferencial e integral: Ciencias sociales y económico administrativas. México: Santillana, 2009.

MOSCHKOVICH, J. Students' interpretations of linear equations and their graphs. En: SPEISER, R.; MAHER, C.; WALTER, C. (Ed.). Proceedings of the 14th Annual Meeting of the International Group for the Psychology of Mathematics Education, Snowbird: North American Chapter, 1990. p. 109-116.

MUDALY, V.; MOORE-RUSSO, D. South African teachers' conceptualisations of gradient: A study of historically disadvantaged teachers in an Advanced Certificate in Education Programme. Pythagoras, Durbanville, v. 32, n. 1, p. 27-33, 2011.

NAGLE, C.; MOORE-RUSSO, D. Connecting Slope, Steepness, and Angles. Mathematics Teacher, Reston, v. 107, n. 4, p. 272-279, 2013a.

NAGLE, C; MOORE-RUSSO, D. The concept of slope: Comparing teachers' concept images and instructional content. Investigations in Mathematics Learning, London, v. 6, n. 2, p. 1-18, $2013 \mathrm{~b}$.

NAGLE, C.; MOORE-RUSSO, D. Slope across the Curriculum: Principles and Standards for School Mathematics and Common Core State Standards. The Mathematics Educator, Georgia, v. 23, n. 2, p. 
40-59, 2014.

NAGLE, C.; MOORE-RUSSO, D.; MARTINEZ-PLANELL, R. A framework for describing conceptions of slope. En: WOOD, M. B.; TURNER, E.; CIVIL, M.; ELI, J. L. (Ed.). Proceedings of the 38th annual meeting of the North American Chapter of the International Group for the Psychology of Mathematics Education, Tucson: The University of Arizona, 2016. p. 195-198.

NAGLE, C.; MOORE-RUSSO, D.; STYERS, J. L. Teachers' Interpretations of Student Statements about Slope. En: GALINDO, E.; NEWTON, J. (Ed.). Proceedings of the 39th annual meeting of the North American Chapter of the International Group for the Psychology of Mathematics Education, Indianapolis: Hoosier Association of Mathematics Teacher Educators, 2017. p. 589-596.

NAGLE, C.; MOORE-RUSSO, D.; VIGLIETTI, J.; MARTIN, K. Calculus students' and instructors' conceptualizations of slope: a comparison across academic levels. International Journal of Science and Mathematics Education, Taipei, v. 11, n. 6, p. 1491-1515, 2013.

NOBLE, T.; NEMIROVSKY, R.; WRIGHT, T.; TIERNEY, C. Experiencing change: The mathematics of change in multiple environments. Journal for Research in Mathematics Education, Reston, v. 32, n. 1, p. 85-108, 2001.

PLANINIC, M. et al. Comparison of student understanding of line graph slope in physics and mathematics. International Journal of Science and Mathematics Education, Berlin, v.10, p. 1393$1414,2012$.

RASSLAN, S.; VINNER, S. The graphical, the algebraical and their relation: The notion of slope. En: MEIRA, L.; CARRAHER, D. (Ed.). Proceedings of the 19th Conference of the International Group for the Psychology of Mathematics Education, Recife: Brazil, 1995. p. 264-271.

SALAZAR, V. Matemáticas 3. México: Nueva Imagen, 2010.

SCHOENFELD, A. H.; SMITH, J. P.; ARCAVI, A. Learning: The microgenetic analysis of one student's evolving understanding of a complex subject matter domain. En: GLASER, R. (Ed.). Advances in Instructional Psychology, Hillsdale: Lawrence Erlbaum Associates, 1993. p. 55-175.

SEP. Matemáticas III. Serie programas de Estudios. México, D. F.: Secretaría de Educación Pública, 2013. Disponible en: $<$ http://www.dgb.sep.gob.mx/02-m1/03-iacademica/01programasdeestudio/3er_SEMESTRE/Matematicas_III_biblio2014.pdf $>$. Acceso en: 20 de Mayo de 2018.

STANTON, M.; MOORE-RUSSO, D. Conceptualizations of Slope: A Review of State Standards. School Science and Mathematics, Hoboken, v. 112, n. 5, p. 270-277, 2012.

STROUP, W. Understanding qualitative calculus: A structural synthesis of learning research. International Journal of Computers for Mathematical Learning, Wayshesville, v. 7, n. 2, p. 167$215,2002$.

STUMP, S. Developing preservice teachers' pedagogical content knowledge of slope. The Journal of Mathematical Behavior, v. 20, n. 2, p. 207-227, 2001.

STUMP, S. Secondary mathematics teachers' knowledge of slope. Mathematics Education Research Journal, Basel, v. 11, n. 2, p. 124-144, 1999.

TEUSCHER, D., Y REYS, R. Rate of Change: AP Calculus Students "Understandings and Misconceptions after Completing Different Curricular Paths. School Science and Mathematics, Hoboken, v. 112, n. 6, p. 359-376, 2012. 
TEUSCHER, D.; REYS, R. Slope, rate of change, and Steepness: Do students understand the concepts? Mathematics Teacher, Reston, v. 3, n. 7, p. 519-524, 2010.

YERUSHALMY, M. Designing representations: Reasoning about functions of two variables. Journal for Research in Mathematics Education, Reston, v. 28, n. 4, p. 431-466, 1997.

ZASLAVSKY, O.; SELA, H.; LERON, U. Being sloppy about slope: The effect of changing the scale. Educational Studies in Mathematics, Dordrecht, v. 49, p. 119-140, 2002.

Submetido em 13 de Novembro de 2018. Aprovado em 28 de Junho de 2019. 


\section{Anexo}

\section{Tareas de la Entrevista}

Tarea 1. Obtenga la pendiente de la recta $l_{1}$ de todas las formas que usted conozca.

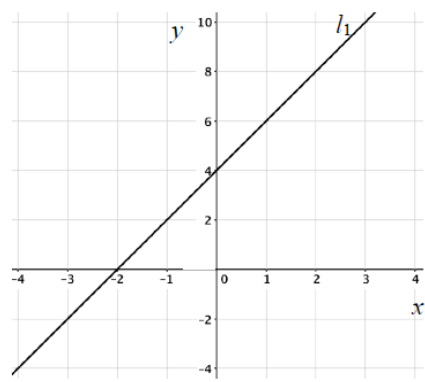

Tarea 2. ¿Cómo son entre sí las pendientes de los techos A, B y C?

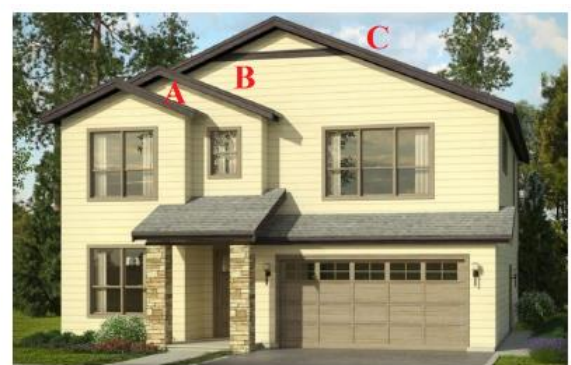

Tarea 3. Construya la gráfica la recta que pasa por el punto $\mathrm{A}(1,-2)$ y cuya pendiente sea 3 . ¿Es posible que existan otras rectas que tengan la misma pendiente? Argumente.
Tarea 4. Identifique la tabla de valores que proviene de una función lineal y argumente su elección.

\begin{tabular}{|c|c|c|c|c|c|}
\hline$x$ & $F(x)$ & $x$ & $F(x)$ & $\mathbf{x}$ & $F(x)$ \\
\hline 1 & 1 & 1 & 2 & -2 & 6 \\
\hline 2 & 4 & 2 & 4 & -1 & 3 \\
\hline 3 & 9 & 3 & 6 & 0 & 0 \\
\hline 4 & 16 & 4 & 8 & 1 & 3 \\
\hline
\end{tabular}

Tarea 5. La profesora presentó la gráfica de dos rectas y pidió a sus alumnos que mencionaran al menos una diferencia entre ellas. Carla señaló que la pendiente de $l_{1}$ es mayor que la pendiente de $l_{2}$, al respecto, Juan comentó que no compartía la opinión de Carla. a) ¿Qué cree que podría estar pensando cada alumno?

b) ¿Quién cree que tenga la razón y por qué?
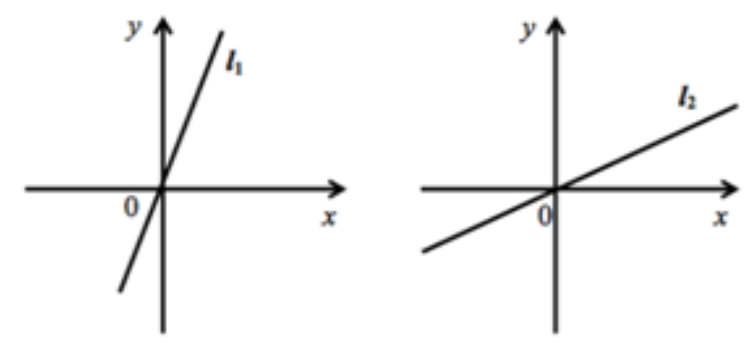

Tarea 6. En Italia se encuentra una subida conocida como el "Salita Scanuppia" en la que una señal de tráfico advierte claramente lo que se avecina, en esta aparece un $45 \%$ tal como se ve en la imagen 1, ¿Qué significa esa señal?

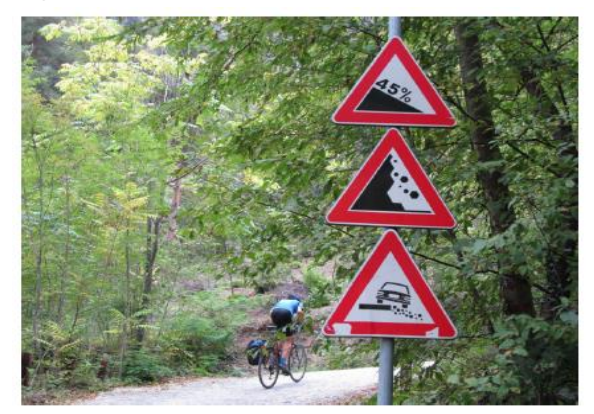

Imagen 1.Salita Scanuppia 
Tarea 7. María tiene una cantidad de dinero ahorrada en el banco. De su salario, guarda la misma cantidad quincenal. Después de tres quincenas, ella tiene ahorrado $\$ 2100$. En la séptima quincena su ahorro es de $\$ 2900$.

a) ¿Cuánto ahorra María cada quincena?

b) ¿Cuánto tenía inicialmente en el banco?

Tarea 8. Analice las cuatro gráficas y responda, ¿cuáles podrían tener una pendiente de 2? Argumente su elección y escriba por cada inciso su análisis.

a)

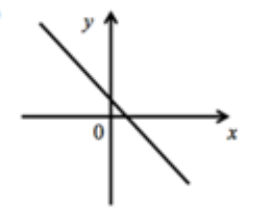

c)

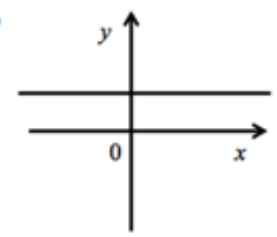

b)

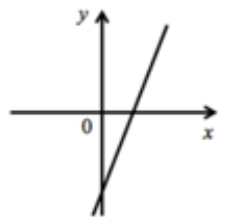

d)

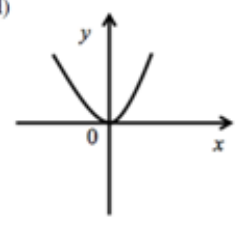

Tarea 9. Analice las ecuaciones dadas y responda lo que se pide
I. $y=2 x$
II. $y=-\frac{1}{2} x$
III. $y=4 x$
IV. $y=3$
V. $y=-3 x+2$
VI. $y=2 x+4$
VII. $y=3 x+1$

a) Elija y escriba las ecuaciones que se corresponden con rectas crecientes, decrecientes, constantes. Argumente su respuesta.

b) Elija y escriba las ecuaciones que representan rectas paralelas entre sí. Argumente su respuesta.

c) Elija y escriba las ecuaciones que representan rectas perpendiculares entre sí. Argumente su respuesta.

d) Elija y escriba las ecuaciones que representan rectas cuya pendiente es dos.

Tarea 10. La recta $k$ forma un ángulo de $30^{\circ}$ con el eje $x$, y la recta $l$ forma un ángulo de $60^{\circ}$ con el eje $x$. Luis asegura que la pendiente de la recta $l$ es el doble de la pendiente de la recta $k$. ¿Qué opina sobre la afirmación del estudiante?

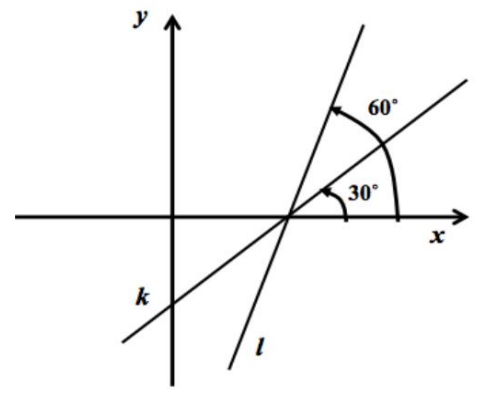

Tarea 11. Dada la gráfica de la recta $l$. Construya al menos dos rectas, que tengan la misma pendiente. Argumente su respuesta

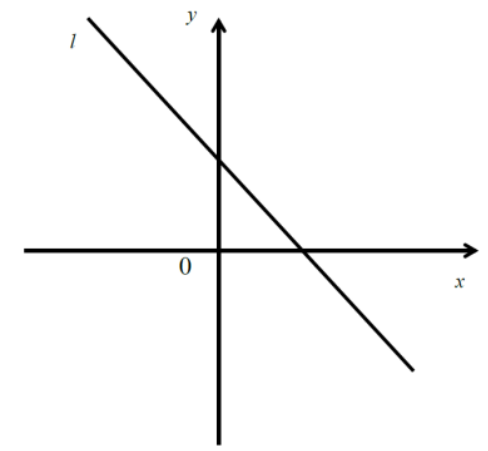

